\title{
Impact of Drones on Precision Agriculture
}

\author{
Will Smith ${ }^{1}$ and Peng Chan ${ }^{2+}$ \\ ${ }^{1}$ California Coast University and DroneData, LLC, USA \\ ${ }^{2}$ California State University-Fullerton, USA
}

\begin{abstract}
This study seeks to examine the use of drones and their impact on precision agriculture. The rapid expansion of the world's population has given rise to increasing crop scarcity. This, in turn, has necessitated the use of agricultural automation whereby unmanned aircraft systems (UAS), more commonly known as drones, can serve as a pivotal data gathering device. Beyond the advantages of accessibility, drones are capable of providing near-real-time remote sensing data from either the field or from the farm home base with immediate uplink capabilities for analytical processing. The immediate accessibility of drone imagery data and the corresponding analytical findings would allow farmers to respond quickly to operational changes. The disruptive force of this unprecedented data-capturing device lies in converting the data into useful and invaluable information for farmers. Due to the accessibility and cost effectiveness of drones to fly at low altitudes on daily frequencies, combined with real-time crop diagnosis through image recognition, one could expect to see drones playing a bigger role in preventive precision agriculture (PPA).
\end{abstract}

Keywords: Drones, Data Gathering, Analytics, Intelligence Machines, Precision Agriculture.

\section{Introduction}

The "Basic Needs" approach introduced by the International Labor Organization at the World Employment Conference in 1976 referred to a list of life's most essential requirements - food (including water), shelter, and clothing. Additional lists of today have come to tack on sanitation, education, and healthcare. However, more than four decades later, satisfying just the world's food needs remains a challenge in an agribusiness market that has a massive $\$ 5$ trillion economic, social, and environmental footprint (Goedde et al, 2015). This challenge opens and nurtures pathways to investment throughout the value chain.

Despite productivity achievements that delivered more than adequate food supplies in parts of the world, industry experts contend that if current trends carry on and if left unaddressed, then by 2050, crop scarcity for human consumption is very likely. Advancements in precision agriculture have occurred through the use of crop yield monitors on GPS-equipped combine harvesters as well as the use of greater variable rate technology (VRT) applications like seeders and sprayers. Although incremental increases in crop productivity have been achieved, meeting the world's food supply demand will require disruption of the current trends (Goedde et al, 2015).

As GPS and VRT technologies have brought early improvements to agribusiness, the question is: will data gathered from drones using revolutionary analytical techniques unlock a potential boundless opportunity for farmers to reduce their cost of goods sold? Tractica, a market intelligence firm that specializes in human interaction with technology, predicted drone applications to grow from $\$ 3$ billion to $\$ 74$ billion by 2024 (Tractica, 2016). Increasing crop scarcity is fueling the rapid necessity of using agricultural automation in which drones serve as an indispensable data gathering device.

\footnotetext{
${ }^{+}$Corresponding author. Tel.: + 1-6268078887.

E-mail address: pengchan@gmail.com.
} 
The purpose of this study is to examine the impact drones are making in precision agriculture. The research further seeks to reveal the greater value of UAV technology within the analysis of the agricultural crop field data.

\section{Background}

The conceptual theory to apply accurate and precise amounts of agricultural inputs of crop stimuli such as water, fertilizer, and pesticides at the correct and exact time as a means to maximize crop yields has come to be known as "Precision Agriculture." Developing from this theory was an early form of crop field grid sampling to generate primitive input recommendation maps.

When the "Information Age" arrived, global positioning systems (GPS) radically improved crop field zone mapping by enabling the farmer to vary the rate of fertilizer disseminated across a field that would otherwise have been placed in areas that did not require the fertilizers. This incremental step toward improving crop yields while optimizing agricultural inputs unambiguously demonstrates precision agriculture as the name implies. Seeking to continuously apply the right amount of agricultural inputs to the right crop field location and at the precise moment improves crop health, soil vitality and groundwater concentration, all of which benefit the entire crop life cycle. For this reason, precision agriculture is crucial to achieving overall sustainable agriculture.

\section{Pursuit of Sustainability}

Merely meeting global food demands on an annual incremental basis is not enough, longer term sustainability requires high-tech solutions to fuel agricultural automation. Agribusiness welcomes the unmanned aircraft systems (UAS) more commonly known as drones to the equation as the disruptive technology solution singling promising benefits. The United States Federal Government in 2012 commissioned the Federal Aviation Administration (FAA) to deliver a solution on how best to integrate drones into the National Airspace System (NAS). Despite scores of uses for UAS, the commissioned study concluded that precision agriculture and public safety were the most promising markets with a ninety (90\%) percent usage factor (AUVSI, 2013).

Given the unprecedented data gathering capabilities of drones as a result of ease of use and connectivity with cloud-based data analytics the contemplated agribusiness use targets included crop surveillance thus virtually eliminating manual walking of fields, remote sensing, aerial photography, videography, photo mapping, soil map creation, crop health condition monitoring, spraying of chemicals, crop dusting, and materials protection. The FAA envisioning the monumental and rapid growth of the UAS market because of scores of alternative uses identified in the 2012 study, regulatory efforts to safeguard the national airspace system (NAS) were accelerated starting with the issuance of the Operation and Certification of Small Unmanned Aircraft Systems Notice of Proposed Rulemaking (NPRM) (Federal Aviation Administration, 2015).

\section{Technology Science}

The economics of UAS technology allows for drones to form part of traditional farming equipment accessible immediately by the farmer. Beyond the benefits of accessibility, drones are capable of providing near-real-time remote sensing data from either the field or from the farm home base with immediate uplink capabilities for analytical processing. This is the opposite of traditional satellite and piloted aircraft-based imagery which creates overlong lag times. The immediate accessibility of drone imagery data and the corresponding analytical findings empower the farmer to implement the biggest operational changes.

Equipped with advanced real-time sensors measuring everything from chlorophyll levels to plant water status with hyperspectral and multispectral imaging capabilities by which Normalized Difference Vegetation Index (NDVI) maps can be generated, drones give farmers new ways to increase yields and reduce crop damage. Using the science of photogrammetry, lines of sight (also known as rays owing to their optical nature) photographs taken by drones at various locations through triangulation are mathematically intersected to produce the three-dimensional coordinates of the points of interest. This combining of science 
with technology on the drone image data benefits farmers in three ways: first, seeing a crop from the air can reveal patterns that expose everything from irrigation problems to soil variation and even pest and fungal infestations that are not apparent at eye level; second, airborne cameras mounted on these drones can take multispectral images, capturing data from the infrared as well as the visual spectrum, which can be combined to create a view of the crop that highlights differences between healthy and distressed plants in a way that the naked eye cannot see; and third, a drone can survey a crop every week, every day, or even every hour (Anderson, 2014). These pictures when combined create a time series animation of imagery, which can show changes in the crop, revealing trouble spots or opportunities for better crop management.

Another science, geomorphology, refers to the attempt by scientists to understand landscape appearances by studying the origin and evolutionary topographic and bathymetric characteristics resulting from certain processes occurring near the Earth's surface. Bringing this science together with spatial data and geographic information science (GIS) the offshoot is a better understanding of landforms, vegetation, and the relationships between plants and terrain properties. This data is very useful in carrying out better precision agriculture.

As science and technology merge devices such as drones, which make for great data gathering, have emerged. To that end, precision agriculture is more than merely the collection of data; it is what is done with the data that is important.

\section{Drone Data Analytics}

While drones that are equipped with sensors and cameras capture multitudes of data, it is only when correctly configured and aligned with analytical system processes that farmers can detect pests, plant diseases, invasive weed growth, irrigation problems, and soil erosion; otherwise, it is simply data to farmers. The disruptive aspect of this unprecedented data capturing device lies in converting this data into useful and invaluable information which farmers can deploy. Combining aerial crop photos with image processing software, farmers can transform pictures into one large orthomosaic image, then applying agribusiness algorithms like Normalized Difference Vegetation Index (NDVI) to create a reflectance map of a crop field. An example of image processing software is Pix4Dmapper Ag (Pix4) which is a powerful program that transforms images into geo-referenced index maps. With complete mobility to manually scout and walk crop fields, Pix4 allows farmers to match NDVI with ground observations using any GPS-enabled device to digest the data and allow easy to view results. Figure 1 is an example of this output imagery. The green areas signify higher NDVI values reflective of strong vegetation, while the red areas depict lower values and weaker vegetation.

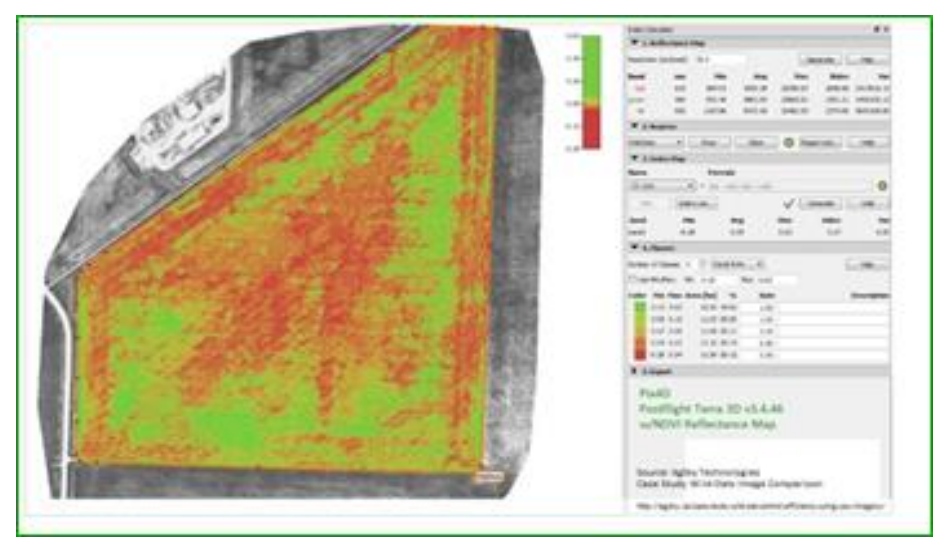

Fig. 1: "Source; AgSky Technologies Case Study 'Wild Oats Image Comparison"

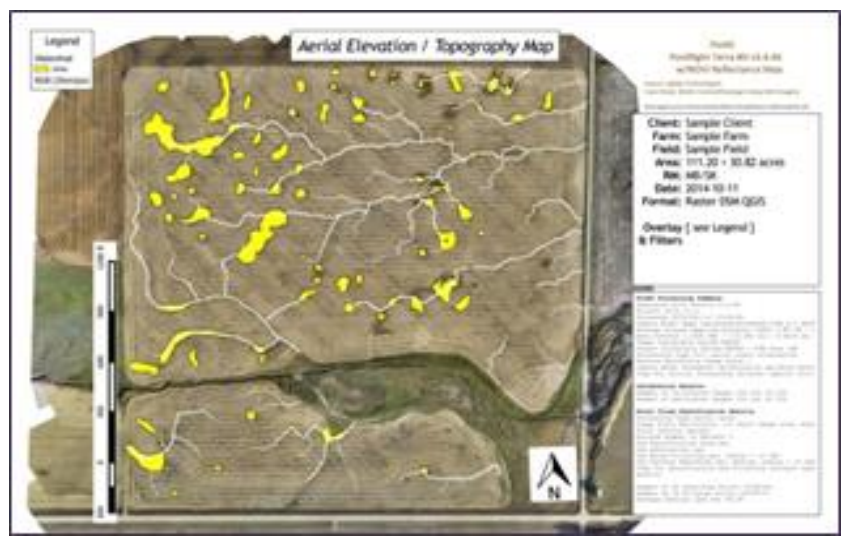

Fig. 2: "Source; AgSky Technologies Case Study "Water Control/Drainage Using"

This Pix4 multipurpose software program using drone-sourced elevation data with the fill sinks algorithm can further identify irrigation and drainage detriments on crop fields (Wang \& Liu, 2006). In October 2014 AgSky Technologies in eastern Saskatchewan conducted a drone flight that captured 336 images across a 160 -acre crop field for the purpose of water and drainage control irrigation improvements (Genik, 2014). The image data underwent approximately four hours of accelerated visual compute processing to generate a $1 \mathrm{gb}$ orthomosaic with an $830 \mathrm{mb}$ digital surface map (DSM) providing a geo- 
referenced triangulated elevation map of the field. Combining these data files resulted in a $12.6 \mathrm{gb}$ detailed 3D point cloud with an estimated 74 million data points. Linking this data directly to the farmers GPS equipped tractor permitted prompt and efficient installation of the necessary drainage system to the exact location. Figure 2 illustrates the specificity of this imagery resolution with the yellow areas signaling watershed areas.

\section{Drone Data Impact}

As plant disease and irrigation remain problems, soil erosion is arguably a tremendous threat, especially in tropical areas where high rainfalls are plentiful year round. Illustrating the attention UAS have captured from agribusinesses, Dole Philippines, a subsidiary of the fruit production giant Dole Food Company, has been flying drones daily in its plantation fields. Dole has utilized drone-based imagery to create crop field mapping outputs as a guide in designing new topographic terrain adapted field layouts to reduce erosion on these plantations (King, 2016).

Drone data outputs are now tapping into artificial intelligence (AI) deep learning as crop diseases remain the major threat to food sustainability. Through a collaborative effort of many institutes that included Switzerland's School of Life Science and the Center for Infectious Disease Dynamics of Penn State University, a study compiled in April 2016 demonstrated the technical feasibility to render crop diagnosis through photographic image recognition. Using deep learning, a subfield of computer science where algorithms can learn from and make predictions on data, from 54,306 images of diseased and healthy plant leaves, a convolutional neural network powered by NVIDIA Tesla K40 GPUs was used to classify both crop species and disease status of 38 classes with over 99\% accuracy (Mohanty et al, 2016).

NVIDIA, viewed as the AI computing company of the Intelligent Industrial Revolution, is tackling humanity's food supply challenge by powering AI technologies to accelerate greater precision agriculture. From this study, researchers have launched "Plant Village", an open access database of 50,000+ images of healthy and diseased crops, with the objective to help farmers around the world by providing them with the ability to send a photo of their diseased plant, after which the application will automatically diagnose through a return communication (NVIDIA, 2016).

\section{Conclusion}

As drone devices provide greater and faster data capturing capabilities, analytical processing leveraging photogrammetry will soon be connected real-time to a fully automated self-learning AI crop diagnosis resource center, which will take precision agriculture to a preventive level. The accessibility and cost effectiveness of drones to fly at low altitudes on daily frequencies, coupled with real-time crop diagnosis through image recognition, are likely to promote the use of Preventive Precision Agriculture (PPA) in agribusiness.

\section{References}

[1] Goedde, L., Horii, M., \& Sanghvi, S. (2015). Pursuing the global opportunity in food and agribusiness. McKinsey \& Company. Retrieved from http://www.mckinsey.com/industries/chemicals/our-insights/pursuing-the-globalopportunity-in-food-and-agribusiness

[2] Tractica (2016). Agricultural Robots: Driverless Tractors, Unmanned Aerial Vehicles, Material Management, Field Crops and Forest Management, Soil Management, Dairy Management, and Animal Management for Precision Agriculture. Research Report, 4th Quarter 2016. Retrieved from https://www.tractica.com/research/agricultural-robots/

[3] Association for Unmanned Vehicle Systems International [AUVSI]. (2013). The Economic Impact of Unmanned Aircraft Systems Integration in the United States. Retrieved from https:/higherlogicdownload.s3.amazonaws.com/AUVSI/958c920a-7f9b-4ad2-9807f9a4e95d1 ef1/UploadedImages/New_Economic\%20Report\%202013\%20Full.pdf

[4] Federal Aviation Administration (2015). FAA Aerospace Forecast Fiscal Year 2016-2036. FAA Data Research Aviation Aerospace. Retrieved from 
https://www.faa.gov/data_research/aviation/aerospace_forecasts/media/FY2016-36_FAA_Aerospace_Forecast.pdf

[5] Anderson, C. (2014). Agricultural drones relatively cheap drones with advanced sensors and imaging capabilities are giving farmers new ways to increase yields and reduce crop damage. MIT Technology Review. Retrieved from https://www.technologyreview.com/s/526491/agricultural-drones/

[6] Wang, L. and Liu, H., (2006). An efficient method for identifying and fill surface depressions in digital elevation models for hydrologic analysis and modelling. Int. J. Geogr. Inf. Sci., 20, 193-213.

[7] Genik, W. (2014). Case Study: Water control/drainage using UAV imagery. AgSky Technologies. Retrieved from http://agsky.ca/case-study-water-control-drainage-using-uav-imagery/

[8] King, N. (2016). Case Study; World's Largest Pineapple Plantation Part 1: Reducing Soil Erosion. Pix4D. retrieved from https://pix4d.com/worlds-largest-pineapple-plantation-part-i-reducing-soil-erosion/

[9] Mohanty, S., Hughes, D., \& Salathe, M. (2016). Using Deep learning for Image-Based Plant Disease Detection. Cornell University Library. Retrieved from https://arxiv.org/ftp/arxiv/papers/1604/1604.03169.pdf

[10] NVIDIA Developer (2016). Artificial Intelligence Helping to Ensure Humanity's Future Food Supply. NVIDA News Center Research. Retrieved from https://news.developer.nvidia.com/artificial-intelligence-helping-to-ensurehumanitys-future-food-supply 